Marta ZUBACZ

Ateneum-Szkoła Wyższa w Gdańsku Maurizio BONINO ${ }^{1}$

Ateneum-Szkoła Wyższa w Gdańsku

\title{
La leggenda del regno del Prete Gianni
}

\begin{abstract}
Summary
The reign of Prester John (Prete Gianni), one of the most interesting legends of the Middle Ages, can ignite the imagination even today. It is not surprising that the eyes and hearts of the monarchs and popes were facing east, where they sought their legendary kingdom characterized by unimaginable power, wealth, miracles and at the same time a pure Christian life.
\end{abstract}

Keywords: the legend of Prester John, Prester John, Prete Gianni, John the Presbyter

\section{La lettera del prete Gianni}

\subsection{Le origini della leggenda - chi era il Prete Gianni?}

Il regno del Prete Gianni, una delle leggende più interessanti del Medioevo, può accendere l'immaginazione anche oggi ma nel passato più che mai. Non c'è da stupirsi che gli occhi e il cuore dei monarchi e dei papi fossero rivolti a est, dove si cercava il suo leggendario regno caratterizzato da potere inimmaginabile, ricchezza, miracoli e al tempo stesso una pura vita cristiana.

I primi riferimenti al misterioso stato risalgono al XII secolo, quando rapporti anonimi di gerarchi indiani vennero pubblicati a Roma e a Costantinopoli durante il pontificato di papa Callisto II. Questi viaggi probabilmente non ebbero mai luogo, ma certamente hanno sempre suscitato un forte interesse per questa terra lontana. La prima testimonianza scritta su Prete Gianni ci giunge dalla cronaca di Otto von Freising, che era presente a Viterbo quando Papa Eugenio III ricevette la legazione del sovrano di Antiochia, Raimondo, il quale chiedeva aiuto nella lotta contro i musulmani in Terra Santa e menzionava un potente alleato, Gianni, che non era solo un re, ma anche un prete il quale era pronto a sostenere le crociate, ma le acque impetuose del fiume Tigri glielo avevano impedito:

Narrabat etiam, quod ante non multos annos Iohannes quidam, qui ultra Persidem et Armeniam in extremo oriente habitans rex et sacerdos cum gente sua Christianus est, sed Nestorianus, Persarum et Medorum reges fratres, Samiardos dictos, bello petierit atque Ebactani, cuius supra mentio

1 https://orcid.org/0000-0002-6355-1585. 
habita est sedem regni eorum expugnaverit. Cui dum prefati reges cum Persarum, Medorum, Assyriorum copiis occurrerent, triduo utrisque mori magis quam fugere volentibus, dimicatum est. Presbyter Iohannes - sic enim eum nominare solent - tandem versis in fugam Persis cruentissima cede victor extitit. Post hanc victoriam dicebat predictum Iohannem ad auxilium Hierosolimitanae ecclesiae procinctum movisse, sed, dum ad Tygrim venisset ibique nullo vehiculo traducere exercitum potuisset, ad septentrionalem plagam, ubi eundem amnem hiemali glacie congelari didicerat, iter flexisse² ${ }^{2}$

La credenza nell'esistenza di questo potente stato accrebbe quando lo stesso Gianni scrisse nel 1165 una lettera all'imperatore bizantino Manuele I Comneno. In quel periodo la convinzione dell'esistenza di questo regno era così diffusa che persino papa Alessandro III nel 1177 mandò lì il suo emissario Filippo con una legazione a cercare codesto regno ma di essa si persero le tracce e nessuno seppe mai cosa gli fosse successo. Gianni era considerato come un alleato nella lotta contro i musulmani per cui non c'era di meravigliarsi che si cercasse costantemente di trovarlo. Allearsi con un sovrano così forte avrebbe certamente spostato le sorti della guerra dalla "parte giusta". Si cominciò realmente a credere nella leggenda che i crociati durante l'assedio di Diametta nel 1219, non attesero l'aiuto di Federico II e andarono al Cairo sperando nell'aiuto di Gianni. Questo fatto è registrato nella cronaca di Radulphus de Coggeshall:

Rumores per totam Christianitatem circumquaque dispersi sunt, quod Rex David, cognomento Joannes presbyter, de India cum magno exercitu adveniens, Persidem et Mediam, et multas alias terras et provinicias Sarracenorum, sibi subjugaverat; et quod mandaverat Caliphae de Baldac, summo Papae Sarracenorum, quod eum debellaturus esset atque totum paganismum, nisi se ad fidem Christi convertissent; atque exercitui christianorum apud Damietam et in terra Jerosolymitana celerem subventionis succursum esset praestiturus ${ }^{3}$.

Naturalmente, l'aiuto "di David" non arrivò mai.

2 Ottonis Episcopi Frisigensis Chronica sive historia de duabus civitatibus. Liber VII 365-366, in Scriptores Rerum Germanicarum in usum scholarum ex monumentis Germaniae historicis separtim editi. Hannoverae et Lipsiae Impensis Bibliopolii Hahniani 1912.

${ }^{3}$ Radulphi de Coggeshall Chronicon anglicanum, De expugnatione Terræ Sanctæ libellus, Thomas Agnellus De morte et sepultura Henrici regis Angliæ junioris, Gesta Fulconis filii Warini, excerpta ex Otiis imperialibus Gervasii Tileburiensis. Ex codicibus manuscriptis edidit Josephus Stevenson 1875r., pag. 190. 
Un esempio interessante della fede universale nel potente Gianni è senza dubbio il fatto che nel 1221 il vescovo di Antiochia, Jacob de Vitry, riferì nella sua lettera a Papa Onorio III che l'esercito dal sommo sacerdote Gianni: "Excerpta de historia gestorum David regis Indorum qui presbyter Iohannes a vulgo appellatur"4, che si trovava a due settimane da Antiochia, attese a muoversi verso Gerusalemme occupata dai musulmani.

La credenza dell'autenticità della missiva di Gianni era così forte che, dopo l'invenzione della stampa, questa lettera era ancora diffusa e tradotta in molte lingue e l'era delle scoperte geografiche portò a cercare il regno in nuove direzioni.

Chi era, tuttavia, il misterioso Gianni? Il nome stesso non è inequivocabile. La leggenda di Gianni ha raggiunto tutti gli angoli dell'Europa. Si credeva che egli fosse il discendente di San Tommasso (l'Incredulo), che secondo la tradizione, era andato a predicare il Vangelo in India, infatti: "VI.I. Coronatio Thomae apostoli et Misdaeus rex Indiae, Iohannes eius filius huiusque mater Tertia"5; per altri era il discendente di uno dei Magi venuti a Betlemme per adorare Gesù come nel caso di Giovanni da Hildesheim ${ }^{6}$, tra i cristiani che vivevano nei territori sotto il controllo dei crociati il Re Gianni veniva identificato nel Gengis-Khan. Con questa ultima interpretazione era d'accordo Giacomo da Vitry, tuttavia a questa teoria si opponevano Marco Polo e Guglielmo da Rubruck: il primo nel "Milione" descriveva il Prete come il sovrano dei Tatari fino al giorno in cui scelsero come sovrano Gengis-Khan. Nel suo libro infatti leggiamo: "Egli è vero ch'egli none aveano signore ma faceano reddita a uno signore, che vale a dire in francesco Preste Gianni, e di sua grandezza favellava tutto l'mondo. Li Tatari li davano d'ogni 10 bestie l'una."7; mentre il secondo nella sua relazione di viaggio, indentificava anche il Prete con il sovrano dei potenti Keraiti:

Et in quadam planicie inter illas alpes erat quidam nestorius, pastor potens et dominus super populum qui dicebatur Naiman, qui erant christiani nestorini. Mortuo Coirchan, elevavit se ille nestorinus in Regem et vocabant eum nestoriani regem Iohannem, et plus dicebant de ipso in decuplo quam veritas esset (...). Huic Iohanni erat frater quidam, potens pastor similiter, nomine Unc; et ipse erat ultra alpes ipsorum Caracatai distans a fratre suo spacio trium ebdomadarum, et erat dominus cuiusdam villule que dicitur

\footnotetext{
${ }^{4}$ Giacomo da Vitry, Lettres de Jacques de Vitry (1160/70-1240), évêque de Saint-Jean d'Acre, éd. par R.B.C. Huygens, Leyde, E.J. Brill, 1960, p. 141.

${ }^{5}$ Peeters P. La martyrolge de Rabban Sliba, Analecta Bollandiana XXVII, 1908, pag. 142.

${ }^{6}$ Confronta: Giovanni da Hildesheim , Historia trium regum, cap. XXVII.

${ }^{7}$ Marco Polo, Il Milione, cap. LXIII, Edizioni Studio Tesi 1991, Pag.71.
} 
Caracarum, populum habens sub se, qui dicebantur Crit et Merkit, qui erant christiani nestorini. ${ }^{8}$

La ricerca del vero regno del Presbitero diede origine a nuove direzioni dove cercarlo. E così Gianni fu identificato da Rudbertus negli "Annales Sancti Rudberti Salisburgensis" in Abaqa, secondo re mongolo di Persia9. Anche Vincenzo di Beauvais aveva il suo punto di vista e considerava Gianni il sovrano indiano. Al contrario, Odorico da Pardenone ha descritto il Prete come un sovrano cinese nel suo "De rebus incognitis." Naturalmente si possono trovare tanti altri esempi di citazioni sull'oscuro re cristiano nelle cronache (sia medievali che successive) o nei rapporti dei viaggi lontani.

Nella ricerca nelle ipotesi sul Prete Gianni, si può vedere quanto il grande re abbia stimolato l'immaginazione di sovrani, papi, cronisti, viaggiatori e gente comune, sebbene non sia mai stato veramente trovato, il misterioso Presbitero cercato in molti paesi, ha contribuito a riformulare la storia di tutto il mondo che è creata dalla curiosità umana.

\subsection{I temi della lettera}

Come accennato precedentemente, la storia del leggendario Prete Gianni ha origine nel XII secolo. Ai governanti, ma anche alla gente semplice, giungevano voci di un regno misterioso il cui sovrano era un potente nestoriano: Gianni. Il suo regno non aveva paragoni: era enorme, ricco e straordinario. La gente del Medioevo cercava di immaginare dove potesse essere ma, come mostreremo più avanti, l'immagine che essi avevano del mondo non rendeva certo il compito facile.

L'aiuto giunse da Gianni. Infatti nel 1165 l'imperatore bizantino Manuele I Comneno ricevette una strana lettera, che iniziava con le seguenti parole: "Presbiter Iohannes, potentia et virtute Dei et domini nostri Iesu Christi dominus dominantium, Emanueli, Romeon gubernatori, salute gaudere et gratia ditandi ad ulteriora transire"10. Il contenuto della lettera - nonostante il fatto che riguardasse principalmente l'aspetto religioso - ha rivelato alcuni dettagli su Gianni e il suo regno e sul perché questa epistola fosse stata scritta.

${ }^{8}$ Gulielmo da Rubruck, Itinerarium, cap. XVII, 2-3, in "Prester John: the Legend and its Source" Keagan Brewer, University of Sydney, Australia, Ashgate 2015; pag. 161.

9 Confronta: Annales Sancti Rudberti Salisburgensis in "Monumenta Germaniae Historica"; edidit Georgius Heinricus Pertz; Scriptorum tomus IX, Hannoverae Impensis Bibliopolii Avlici Hahniani MDCCCLI.

10 Friedrich Zarncke, Der Priester Johannes, 1980, Georg Olms Verlag Hildesheim, New York, pag. 64. 
Gianni si presentò come il sovrano dell'India, il più potente di quelli che governavano sulla terra - "dominus sum dominantum"11, il guardiano della tomba di San Tommaso: "La nostra Sovranità si estende sulle tre Indie e dall'India Maggiore, dove riposa il corpo dell'apostolo Tommaso (... $)^{112}$. Avendo egli saputo che l'imperatore aveva intenzione di mandargli un regalo, decise di contattarlo: "Era noto alla Maestà nostra che onoravi la nostra Eccellenza e che avevi avuto notizia della nostra Altezza. Abbiamo inoltre saputo dal nostro emissario che volevi mandarci qualcosa di divertente e piacevole con cui lusingare la nostra Clemenza"13. È interessante notare che Gianni aveva già rivelato le sue idee politiche proprio all'inizio della lettera. Si rivolse a Manuele I Comneno non come a un'imperatore ma come a un governatore ${ }^{14} \mathrm{e}$ lo rimproverò per aver acconsentito che i suoi sudditi, che egli descrisse sprezzantemente come "poveri greci", lo trattassero come un dio, il che evidenzia il suo l'atteggiamento anti-bizantino.

In quanto uomo lo accettiamo volentieri e ti inviamo tramite il nostro emissario qualcosa di nostro, perché intendiamo e desideriamo sapere se con noi segui la vera fede e se in tutto e per tutto credi in Gesů Cristo nostro Signore. Mentre infatti di noi conosciamo la nostra natura di uomo, i tuoi Grechetti Ti credono un dio, ma noi sappiamo che sei mortale e soggetto alla umana caducità ${ }^{15}$.

La lettera conteneva una descrizione molto dettagliata del regno. L'autore si concentrava sulla ricchezza, sulle meraviglie e su tutte le cose straordinarie che erano sotto il suo controllo. E questi beni erano considerevoli: settantadue re gli pagavano il tributo, settantadue province erano a lui sottomesse. Queste province, sebbene non cristiane, gli pagavono un tributo insieme ai loro governanti ${ }^{16}$. Gianni distribuiva l'elemosina ai suoi sudditi cristiani: "Sono un devoto cristiano e ovunque proteggo e sostengo con elemosine i cristiani poveri governati dalla sovranità della mia Clemenza"17.

Lo stato di Gianni descritto nella lettera era una terra di miele e latte fluido ${ }^{18}$. Era abitato non solo da gente, ma anche da creature straordinarie come giganti,

\footnotetext{
${ }^{11}$ Ibidem, pag. 65, traduzione: "signore dei signori".

${ }^{12}$ Zaganelli Gioia, La Lettera del Prete Gianni, Luni Editrice 2000, pag. 53.

${ }^{13}$ Ibdem, pag. 53.

${ }_{14}$ Por. Friedrich Zarncke, Der Priester Johannes, 1980, Georg Olms Verlag Hildesheim, New York, pag. 64.

${ }^{15}$ Zaganelli Gioia, La Lettera del Prete Gianni, Luni Editrice 2000, pag. 53.

${ }^{16}$ Ibidem, pag. 54-56.

17 Ibidem, pag. 55.

${ }^{18}$ Ibidem, pag. 71.
} 
ciclopi, fauni, satiri. La ricchezza proveniva da giacimenti minerari: Gianni aveva enormi depositi di smeraldi, topazi, ametiste e zaffiri. Anche la flora era isolita: in nessun altro luogo cresceva la pianta "assenzio", che aveva proprietà protettive nella lotta contro Satana. I mari del regno erano sabbiosi, i fiumi ricchi di pietre, ma anche i pesci erano copiosi. Il sovrano viveva in enorme palazzo di gioielli, in cui ogni giorno faceva una festa per diecimila ospiti. Gianni era un sovrano saggio e giusto e, sopratutto, un timorato di Dio; il suo esercito doveva fare una grande impressione:

Quando muoviamo in guerra contro i nostri nemici facciamo portare avanti alla nostra persona tredici croci grandi e molto alte, lavorate in oro e in pietre preziose, ciascuna in un carro al posto dei vessilli; ognuna di esse è seguita da 10.000 cavalieri e da 100.000 fanti armati, senza contare quelli che devono occuparsi delle salmerie, dei carri e del trasporto delle vettovaglie dell'esercito. Quando poi cavalchiamo in forma privata, la Maestà nostra è preceduta da una croce di legno non decorata e disadorna d'oro e di gemme, per ricordare in ogni momento la passione di Gesù Cristo nostro Signore, e da un vado d'oro pieno di terra, per provare che la nostra carne tornerà alla sua origine certa, vale a dire alla terra. Un altro vaso d'argento, pieno d'oro, è portato davanti a noi, affinché tutti comprendano che noi siamo signore dei signori. La nostra Magnificenza sovrabbonda e predomina in ogni ricchezza che c'è sulla terra ${ }^{19}$.

Tuttavia, non furono le ricchezze e le magnificenze descritte nella lettera, a fare in modo che papi e imperatori decisero di cercare Gianni. Le promesse di ricchezza e miracoli eccezionali potevano essere allettanti per i viaggiatori, per coloro che cercavano avventure. Il Prete nel suo scritto tentò i sovrani dell'Europa di allora con la frase: "Abbiamo in voto di visitare il Santo Sepolcro con un grandissimo esercito, in quanto si addice alla gloria della nostra Maestà umiliare e sconfiggere i nemici della croce di Cristo ed esaltare il suo nome Benedetto"20. Questa dichiarazione nella situazione politica di quel tempo doveva fare un'impressione considerevole e stimolare le speranze. Nessuno poteva essere indifferente alla proposta di un alleato così potente nella più importante lotta del tempo. Tanto meno il papa e l'imperatore, i quali avevano lo stesso obiettivo: sconfiggere i musulmani.

19 Zaganelli Gioia, La Lettera del Prete Gianni, Luni Editrice 2000, pag. 74-75.

${ }^{20}$ Ibidem, pag. 55. 
La lettera termina con una dichiarazione della grandezza di Gianni, che umilmente prese il titolo di "Prete":

(...)la nostra Sublimità non voglia essere denominata con un nome più degno di quello di prete, la tua saggezza non deve stupirsi. Nella nostra corte vi sono infatti molti ministeriali i quali sono insigniti di un nome e di una funzione più degni, per ciò che attiene alla dignità ecclesiastica, e persino superiori al nostro nell'ufficio divino. Il nostro scalco è infatti primate e re, il nostro coppiere è arcivescovo e re, il nostro ciambellano è vescovo e re, il nostro maniscalco è re e archimandrita e il capocuoco è re e abate. Dunque l'Altezza nostra non ha accettato di essere designata con questi stessi nomi o di essere insignita con gli stessi ordini dei quali la nostra corte è manifestamente piena, e perciò piuttosto per umiltà ha scelto di essere denominata con un nome meno nobile e con un grado inferiore ${ }^{21}$.

La missiva ebbe un notevole successo in Europa. Dalla lettera nacque la leggenda del regno di Prete Gianni. Fu sulla base della lettera che si svilupparono storie fantastiche, che trassero ispirazione da essa, perché combinò influenze bibliche con antichi miti. Vale la pena notare che per gli abitanti dell'impero d'Occidente l'interesse per la lettera era tanto maggiore quanto più ci si allontanava da Constantinopli. Questo fenomeno può essere spiegato dal fatto che i Bizantini sapevano esattamente cosa c'era in Oriente e ne avevano un'immagine diversa. I mercanti che avevano persino raggiunto la Persia incontravano persone provenienti dalla Cina o dall'India e quindi avrebbero avuto informazioni di prima mano sul regno straordinario esistente.

La lettera tradotta in molte lingue nei secoli successivi fu decisiva per l'espansione cristiana dell'Occidente verso l'Oriente. Fino al XVIII secolo, anche se il contenuto della lettera dimostrava miracoli inimmaginabili, la lettera era considerata autentica.

\subsection{Chi furono gli autori del falso?}

La lettera del Prete Gianni è una delle più interessanti falsificazioni di documenti nella storia. La cosa certa è che il contenuto della lettera è stato diffuso per molti secoli. Probabilmente l'autore della missiva non sarà mai determinato (nessuno ha dubbi sul fatto che non fosse un geniale Gianni), ma alcune premesse potrebbero far luce sui possibili circoli in cui potrebbe aver avuto origine. Naturalmente, dare la paternità della epistola è direttamente correlato al motivo della sua creazione e del suo destinatario. Così, al fine di trovare l'autore della lettera, si dovrebbe

${ }^{21}$ Zaganelli Gioia, La Lettera del Prete Gianni, Luni Editrice 2000, pag. 93. 
guardare attentamente al destinatario o meglio i destinatari. La lettera giunse prima a Manuele I Comneno, poi a papa Alessandro III e all'imperatore Federico Barbarossa. Sembra che questi ultimi due destinatari avessero il loro interesse a ricevere una lettera dal grande sovrano cristiano.

In relazione a Manuele I Comneno la cosa più sorprendente è la sfumatura anti-bizantina della lettera. Sebbene fosse stata scritta con un tono gentile, la frase all'Imperatore come "governatore dei Romei"22 o "re dei Grechetti"23 mostrava che la lettera poteva essere nata in una comunità che non simpatizzava con i Bizantini ed era un'ovvia propaganda contro Bisanzio se, effettivamente, si presume che questi termini fossero stati usati nella lettera intenzionalmente. Una supposizione completamente opposta può essere l'ipotesi che la lettera venne scritta in ambienti in cui la politica era un elemento poco conosciuto e che i termini umilianti rivolti all'imperatore furono creati per ignoranza il che sembra improbabile.

Sebbene il regno di Giovanni fosse cercato in Oriente, la lettera non aveva piuttosto "radici orientali". Sembra improbabile che il sovrano dell'Estremo Oriente fosse in grado di comprendere il latino (e la lettera originale fu scritta in tale lingua ${ }^{24}$ ) a un livello così elevato, quindi è una ragionevole congettura che l'epistola fu redatta nell'Europa occidentale. Il numero di riferimenti alla Bibbia e alle credenze ebraiche può anche suggerire che essa venne creata in un ambiente ben versato nella letteratura biblica ed ebraica per cui un monastero o un'abbazia potrebbero essere il probabile luogo di origine della lettera ${ }^{25}$, infatti, nel Medioevo il clero aveva accesso ai libri ed era in possesso di una fluente conoscenza del latino. Pertanto il fatto che la lettera fu scritta in latino e che in essa si proponesse il sostegno alla lotta contro i musulmani ${ }^{26}$, ci fa presupporre che l'epistola sia nata in seno alla Chiesa. Chi avrebbe tratto beneficio dal ricevere una lettera presumibilmente scritta da un potente alleato in una lotta che i cristiani stavano perdendo? L'ipotesi sull'origine in ambito ecclesiastico della lettera sembra molto probabile. È interessante notare che in questo contesto papa Alessandro III fu l'unico a rispondere anche in forma di lettera all'epistola di un potente pretesacerdote. Nel 1177 a Venezia scrisse una lettera che inizia con le seguenti parole: "Alexander episcopus, servus servorum Dei, in Christo filio, illustri et Magnifico

\footnotetext{
22 Zaganelli Gioia, La Lettera del Prete Gianni, Luni Editrice 2000, pag. 53.

${ }^{23}$ Ibidem, pag. 53.

${ }^{24} \mathrm{Ci}$ sono anche affermazioni dei copisti del dodicesimo secolo che, per la lingua originale, indicavano l'arabo o il greco. Confronta: Helleiner Karl F., Prester John's Letter: A Mediaval Utopia, Phoenix 1959, vol. XIII-2, pag. 54.

25 Nowen Charies E., The Historical Prester John, Speculum XXVIII -3, 28 luglio 1953, pag. 435-445.

${ }^{26}$ Zaganelli Gioia, La Lettera del Prete Gianni, Luni Editrice 2000, pag. 55.
} 
Indorum regi (...)"27 Nell'epistola, Alessandro III proponeva a Gianni un'alleanza con la Chiesa romana. Le interpretazioni di questa lettera sono diverse. È certo, tuttavia, che il Papa cercò disperatamente di trovare un alleato nella lotta contro i musulmani e non scrisse una lettera a una figura mitica, bensì a una figura reale e potente. Insieme alla lettera mandò il suo uomo più fidato - il medico Filippo. Sfortunatamente, non sappiamo quale fu l'esito del viaggio del dottore ma una cosa è certa: questi non tornò dalla spedizione e non si ebbero più sue notizie.

Un'altra ipotesi fu fatta dallo storico Karl Helleiner. Egli credeva che il padre della lettera potesse provenire dalla cerchia di Federico Barbarossa ${ }^{28}$, il quale avrebbe tratto beneficio personale nel riceverla: la sua autorità imperiale in occidente sarebbe stata rafforzata e l'influenza di Comneno sarebbe diminuita. Federico I di Hohenstaufen era in costante conflitto con papa Alessandro III. Anche se da un lato questi dichiarò il rispetto per la dignità della Chiesa nello stato, lo fece solo per la promessa di essere incoronato imperatore.

Una visione completamente diversa è presentata dallo storico Mariusz Szulc, che nel suo articolo sull'Etiopia presenta una teoria molto interessante sull'origine della lettera proveniente da questo paese: "La lettera che diffuse in Europa la leggenda del Prete Gianni era falsa, ma molto probabilmente creata da qualcuno che capì quanto fosse grande la ricchezza dell'Etiopia in quel momento"29.

È interessante notare che il problema della paternità della lettera su cui gli studiosi del Medioevo si basano oggi a quel tempo non esisteva realmente: nessuno, infatti, si chiedeva se la lettera fosse autentica o meno, ma la cosa più importante era che il suo contenuto fosse in linea con l'immagine medievale del mondo. E indubbiamente era così. La questione di chi fosse l'autore era secondaria.

Indovinare lo scopo per cui venne scritta la lettera sarebbe molto utile per risalire al suo autore. Sfortunatamente oggi è praticamente impossibile: l'epistola del Prete Gianni potrebbe essere cosi un'opera letteraria, uno scherzo goliardico come un potente strumento di propaganda politica o religiosa.

\footnotetext{
27 Zarncke F. Der Brief des Papstes Alexanders III an der Priester Johannes in Beckingham Ch., Hamilton B. Prester John, the Mongols and the Ten Lost Tribes; Ashgate Publishing Ltd, 1996, pag. 103-112.

${ }^{28}$ Helleiner Karl F., Prester John's Letter: A Mediaval Utopia, Phoenix 1959, vol. XIII-2, pag. 48.

29 https://histmag.org/Tajemnicze-krolestwo-ksiedza-Jana-1406, consultato in 24.01.2019r., propria traduzione.
} 


\section{Il regno del prete Gianni}

\subsection{La collocazione del regno}

La posizione del regno di Gianni è uno dei principali misteri della leggenda: la corretta direzione dell'esplorazione potrebbe aiutare a svelare il mistero dello stato enigmatico. Per poter comprendere i metodi della ricerca dei viaggiatori medievali, bisogna prendere in considerazione il loro punto di vista del mondo un mondo in cui tutto, anche le mappe, è soggetto a Dio. La cultura nel Medioevo "era tenuta" nei monasteri, quindi la comprensione dell'universo era un sguardo attraverso il prisma del Creatore. I cosmografi medievali erano di solito persone associate alla Chiesa che accettavano la Bibbia come fondamento del loro lavoro di ricerca.

La ricerca della potenza del Prete iniziò già nel XII secolo e proseguì nel corso dei secoli. L'Asia era la prima destinazione per messaggeri e viaggitori. Queste spedizioni hanno contribuito a una migliore comprensione delle abitudini delle persone che abitavano questo continente - ma non è stata trovata alcuna prova dell'esistenza di un potente sovrano nestoriano. In Asia i viaggiatori cercavano di trovare uno stato "Tre India", difficile da identificare con un paese concreto. Le ricerche di "Tre India"si svolse in gran parte sulla costa del Malabar, perché era qui che si trovavano gruppi di cristiani: nestoriani e monofisiti e si sapeva che Gianni era un sovrano nestoriano. A causa della mancanza di risultati, l'area di ricerca si spostò in un'area sempre più vasta in Asia - il regno venne ricercato anche a Karakorum e nel deserto del Gobi. Alcuni hanno identificato lo stato del Prete anche con il paese sotterraneo di Shamabal, di cui le monache buddiste raccontavano storie incredibili. Un'intensa ricerca di contatti con il sovrano perduto ebbe luogo che copriva l'attuale Cina, Mongolia, Iran o India. A partire dal XIV secolo spedizioni cambiarono la loro direzione: i viaggiatori si diressero verso l'Africa. Tuttavia nel XV secolo l'esplorazione iniziò anche in America.

L'ultimo, ma il più interessante in termini di storia, sembra essere la ricerca “etiope". L'Etiopia corrispondeva perfettamente alla descrizione del regno - un remoto paese cristiano (sebbene con tradizioni diverse), circonadato da nemici musulmani, esotico e molto ricco. È interessante notare che gli abitanti dell'Abissinia/Etiopia persuasero gli europei che il loro è il regno perduto avevano il loro stesso interesse: cercavano alleati per la lotta contro i musulmani. La missione etiope arrivò nel 1518, prima in Portogallo e poi in altri paesi. Nel 1541, durante le guerre con i musulmani, l'Etiopia ebbe persino l'appoggio dei portoghesi. Sfortunatamante l'Etiopia non poteva dare quello che i governanti 
europei cercavano. L'argomento della storia etiope nella leggenda di Gianni è descritto in modo molto interessante dallo storico Mariusz Szulc ${ }^{30}$.

Nel Medioevo e anche nell'era delle scoperte geografiche, il regno del Prete Gianni fu cercato praticamente in tutte le aree conosciute della terra. Una tale situazione mostra quanto viva fosse la convinzione della sua esistenza e quanto grande fosse il bisogno di trovarlo. Nonostante l'ampia ricerca, il regno non venne stato trovato - come se questo cambiasse la sua posizione:

Man mano che i viaggiatori europei si allontanavano dall'Occidente, il Prete Gianni recedeva verso lontananze sempre più mitiche: dagli Urali alla Persia e all'India, dalla Mongolia alla Cina, all'Indocina e alla Manciuria. Ciò che restava fissa era la strabiliante ricchezza del Prete e la sua volontà di accostarsi alla dottrina di Roma. (...) Poi quando l'Asia sembrò non offrire nuovi appigli alle speranze di trovarvi un valido alleato (...) fu la volta dell'Africa: l'Egitto prima, la Nubia infine l'Etiopia ${ }^{31}$.

Una cosa è innegabile: la ricerca in molti continenti ha contribuito allo sviluppo culturale di tutta l'umanità. E questo indipendentemente dal paese di origine di Gianni, è stato senza dubbio il suo più grande dono per l'Europa.

\subsection{La religione, la ricchezza e le meraviglie nel regno}

Uno degli argomenti chiave per la ricerca del regno di Gianni è il tema religioso. Lo stesso Presbitero dedicò molto spazio a questo argomento nella sua lettera. Le idee religiose del sovrano di uno stato potente ebbero un impatto reale sull'area della ricerca del regno perduto. Nella missiva, il presunto autore descrisse se stesso come un seguace del nestorianesimo, una religione il cui nome deriva dal Patriarca di Constantinopoli, Nestorio. La principale dottrina che separava il nestorianesimo dalla Chiesa Romana era il riconoscimento della duplice natura di Cristo. Secondo Nestorio, Gesù aveva due nature: divina e umana, apparentemente solo unite. Maria era soltanto la madre della natura umana di Cristo. Sebbene il nestorianesimo fosse considerato un'eresia e fosse combatutto dalla Chiesa, questa dottrina si stava diffondendo rapidamente nelle aree orientali. E così gli insegnamenti di Nestorio ebbero seguaci in Asia centrale e orientale, in Persia, in Arabia, in Tibet, in India, in Mongolia e persino in Cina. L'intenso sviluppo del nestorianesimo in questi regioni designò la direzione delle spedizioni alla ricerca del regno misterioso. Se Gianni era un nestoriano, il suo paese doveva

\footnotetext{
30 https://histmag.org/Tajemnicze-krolestwo-ksiedza-Jana-1406 (consultato 20.03.2019).

${ }^{31}$ Marco Polo, Il Milione, A cura di Ettore Camesasca; Milano 1997.
} 
essere situato nelle terre in cui questa dottrina si era diffusa. La religione in cui Giovanni credeva, era importante dal punto di vista della Chiesa Romana la rovina graduale del nestorianesimo associato all'espansione dell'Islam nei territori orientali, rese Giovanni un grande alleato nella lotta contro gli infedeli. Il potente re-sacerdote doveva difendere la religione oppressa.

La religiosità dell'autore della lettera è visibile quasi con le prime parole: "Presbiter Iohannes, potentia et virtute Dei et domini nostri Iesu Christi dominus dominantium (...)"32 Certamente, la scelta della parola "Presbiter" è interessante questo termine significava "il maggiore" nella Chiesa che si occupava della formazione e della catechesi. Si può dire che questa espressione fosse l'equivalente della parola contemporanea "sacerdote". Se aggiungiamo il titolo reale ad esso, otterremo un sovrano praticamente ideale - una persona che è sia un'autorità morale che militare. Tuttavia, Gianni spiegò in modo piuttosto complesso ${ }^{33}$ il motivo dell'uso del titolo "Prete" nella lettera:

Se poi vuoi sapere per quale motivo - visto che il creatore di tutto quanto esiste ha fatto di noi il più potente e glorioso tra tutti i mortali - la nostra Sublimità non voglia essere denominata con un nome più degno di quello di prete, la tua saggezza non deve stupirsi ${ }^{34}$.

Nel testo dell'intera lettera Gianni si presentò come un sacerdote dall'Antico Testamento, cioè una persona che custodiva tanto la morale quanto la legge; mentre mostrava ai destinatari della lettera la sua ricchezza e il suo potere: "Si potes dinumerare stellas caeli et harenam maris, dinumera et dominium nostrum et potestatem nostram"35. Tuttavia, nessuno dovrebbe essere sorpreso da un contrasto tra "l'umile servitore di Dio" e "ricco e potente sovrano" - perché Gianni "governava" il suo paese nel modo più cristiano. E il sovrano sottomesso a Cristo era un buon sovrano, che condivideva volontieri le sue ricchezze con gli altri:

\footnotetext{
32 Friedrich Zarncke, Der Priester Johannes, 1980, Georg Olms Verlag Hildesheim, New York, pag. 64; traduzione: "Io, Prete Gianni, per virtù e potere di Dio e di nostro signore Gesù Cristo, signore dei signori (...)".

${ }^{33}$ Confronta: Zaganelli Gioia, La Lettera del Prete Gianni, Luni Editrice 2000, pag. 93.

34 Zaganelli Gioia, La Lettera del Prete Gianni, Luni Editrice 2000, pag. 93.

35 Friedrich Zarncke, Der Priester Johannes, 1980, Georg Olms Verlag Hildesheim, New York, pag.98; traduzione: "Se tu potessi contare le stelle del cielo e la sabbia del mare, allora potresti misurare i nostri domini e la nostra potenza."
} 
In quanto uomo lo accettiamo volentieri e ti inviamo tramite il nostro emissario qualcosa di nostro, perché intendiamo e desideriamo sapere se con noi segui la vera fede e se in tutto e per tutto credi in Gesù Cristo nostro Signore. (...) Se vorrai venire nei nostri domini faremo di te il più grande e il più degno della nostra casa e potrai godere delle nostre ricchezze e, se poi vorrai andartene, te ne andrai ricolmo di ciò che qui abbonda. Ricordati che devi morire e mai peccherai ${ }^{36}$

Il regno del Prete Gianni era ricco di innumerevoli straordinarietà: mari senza acqua $^{37}$, pepe di alta qualità ${ }^{38}$, sorgenti miracolose ${ }^{39}$, ruscelli insoliti ${ }^{40}$, piante preziose (comprese quelle che proteggevano dagli spiriti maligni), fiumi piene di pietre preziose...

Tra i pagani, in una delle nostre province, scorre un fiume che chiamano Indo. Questo fiume, che sgorga dal Paradiso, distende i suoi meandri in bracci diversi per l'intera provincia e in esso si trovano pietre naturali, smeraldi, zaffiri, carbonchi, topazi, crisoliti, onici,berilli, ametiste, sardonici e molte altre pietre preziose. Nel fiume nasce anche un'erba che chiamano assidio e se qualcuno ne porta su di sé la radice scaccia lo spirito maligno e lo costringe a dire chi sia e di dove venga e il suo nome. Ecco perché, in quella regione, gli spiriti maligni non osano invasare nessuno. In un'altra delle nostre province nasce pepe di ogni qualità, che è poi raccolto e scambiato con grano, con biade, con cuoio e con panni. ${ }^{41}$

Sulle terre di Gianni dal cielo cadeva la manna, con cui Dio nutriva gli Israeliti ${ }^{42}$, e le tribù perdute di Israele vivevano $l_{1}{ }^{43}$. È davvero difficile contare tutte le cose straordinarie che Presbitero menzionò nella sua lettera.

Quando leggiamo la lettera del Prete Gianni è difficile resistere all'impressione che il regno perduto sia un luogo in cui si intrecciano tre mondi: il mondo reale, il mondo della fede e il mondo fantastico. Ricchezza inimmaginabile combinata con pura fede cristiana, situata nel posto più incredibile della terra - così possiamo descrivere brevemente il regno del Prete Gianni.

\footnotetext{
36 Zaganelli Gioia, La Lettera del Prete Gianni, Luni Editrice 2000, pag. 53.

${ }^{37}$ Ibidem, pag. 65.

${ }^{38}$ Ibidem, pag. 107-109.

${ }^{39}$ Ibidem, pag. 59.

${ }^{40}$ Ibidem, pag. 67.

${ }^{41}$ Ibidem, pag. 59.

42 Zaganelli Gioia, La Lettera del Prete Gianni, Luni Editrice 2000, pag. 61.

${ }^{43}$ Ibidem, pag. 67.
} 


\subsection{Gli abitanti del regno}

Certamente la parte più fantastica e diversa del regno di Gianni erano i suoi abitanti. Non potevano essere "persone come tante" - non sarebbero mai state allettanti per quelli che carcavano il Prete. Inoltre i cercatori dovevano autenticare l'esistenza dello stato potente che nessuno aveva mai visto. Naturalmente la combinazione degli strani abitanti del regno del Presbitero è dovuta alle molte generazioni cresciute in culture diverse. Una delle culture che ebbe un enorme impatto sulla formazione delle credenze (non solo all'interno del proprio stato) era la cultura dell'antica Grecia. Ai greci veniva attribuito il merito di credere che centauri o satiri vivessero in India. La gente vedeva l'India come una terra di miracoli ${ }^{44}$ e, come sappiamo, era uno dei luoghi principali per la ricerca del regno. La convinzione che in queste terre esistessero unicorni, sirene, arpie o anche persone con la testa di un cane era comune. Certamente, in gran parte, dobbiamo questa credenza alla mitologia, e la mancanza di prove della non esistenza di queste meravigliose creature implicava la loro esistenza. Un grande contributo allo sviluppo delle credenze in esseri favolosi fu dato anche dalla letteratura braminica - che spesso combinava gli elementi del mondo reale con quello fantastico: dobbiamo ai bramini, ad esempio, i panozi.

Quanto grande fosse la credenza nelle strane creature nella mente delle persone, comprese quelle degli scienziati, può essere dimostrata dal fatto che uno degli autori più letti del V secolo, Marziano Capella, dedicò un capitolo del suo libro all'India e agli esseri viventi, come i giganti ${ }^{45}$. Naturalmente non solo grazie agli antichi abbiamo le storie sulle creature meravigliose: anche i cronisti e gli storici medievali (e di epoche succesive) ebbero una grande influenza sulla diffusione di queste credenze, che non potevano separare la finzione dalla realtà nonostante la visione sempre più consapevole del mondo.

Un'altra questione è il rapporto tra la religione e la creazione divina. Tutti gli esseri, anche i più strani, dovevano essere opere dello stesso Dio. Seguendo questo percorso non sorprende che Gianni abbia menzionato nella sua lettera creature sempre più insolite che nella mente della gente non incutevano paura in quanto anch'esse erano l'opera di Dio, ma piuttosto l'estasi e l'ammirazione per la diversità delle opere del Creatore. Se, quindi, la possibilità di ammirare siffatte meraviglie di Dio era solo nel regno perduto, non sorprende che molti avventurieri lo cercassero. Persino Cristoforo Colombo cedette al fascino della storia e, sebbene inizialmente cercò di essere realista, con il passare del tempo esagerò

\footnotetext{
${ }^{44}$ Confronta: Ctesia di Cnido, Indica, lavoro conservato in frammenti nelle opere degli altri autori.

${ }^{45}$ Confronta: Marziano Capella, De nuptiis Philologiae et Mercurii.
} 
con l'immaginazione e raccontò storie di sirene e amazzoni che vivevano in una terra lontana.

Gianni nella sua missiva dedicò molto spazio agli abitanti del regno ${ }^{46}$.

Nei nostri domini nascono e vivono elefanti, dromedari, cammelli, ippopotami, coccodrilli, metagallinari, cameteterni, tinsirete, pantere, onagri, leoni bianchi e rossi, ori bianchi, merli bianchi, cicale mute, grifoni, tigri, sciacalli, iene, buoi selvatici, sagittari, uomini selvatici, uomini cornuti, fauni, satiri e donne della stessa specie, pigmei, cinocefali, giganti alti quaranta cubiti, monocoli, ciclopi, un uccello chiamato fenice e pressoché ogni tipo di animale che vive sotto la volta del cielo ${ }^{47}$.

Dopo un primo elenco degli abitanti, il Prete menzionò le nazioni straordinarie che vivevano nel suo paese e solo dopo l'introduzione del lettore nel suo mondo insolito, Giovanni fece descrizioni dettagliate dei residenti e delle loro abitudini. Questa sembra essere un'azione letteraria volta a suscitare la curiosità e l'immaginazione del lettore:

Abbiamo altre genti che si cibano solo di carne, tanto degli uomini quanto degli animali bruti e dei feti e che non temono la morte. Quando qualcuno dei loro muore, sia i consanguinei che gli estranei lo mangiano con grande avidità e dicono: «É atto santissimo mangiare carne umana». Queste genti si chiamano: Gog e Magog, Amic, Agic, Arenar, Defar, Fontineperi, Conei, Samante, Agrimandi, Salterei, Armei, Anofragei, Annicefelei, Tasbei, Alanei. Proprio queste, insieme a molte altre generazioni, il giovane Alessandro Magno, re dei Macedoni, rinchiuse tra monti altissimi, verso settentrione ${ }^{48}$.

Vale la pena notare, che l'autore della lettera in primo luogo nella sua lista menzionò "Gog e Magog". Considerando l'intera construzione della missiva, non è una scelta causale. Sia Gog che Magog appaiono nella parte apocalittica della Bibbia, ma hanno anche la loro parte non solo nel ciclo siriano delle leggende su Alessandro Magno: da qui il riferimento: "Proprio queste, insieme a molte altre generazioni, il giovane Alessandro Magno, re dei Macedoni, rinchiuse tra monti altissimi, verso settentrione" 49 , ma anche nella letteratura escatologica araba

\footnotetext{
${ }^{46}$ Parlando degli abitanti il re si riferiva anche a tutte le creaturemitiche, ai mostri, agli animali e non solo agli esseri umani.

47 Zaganelli Gioia, La Lettera del Prete Gianni, Luni Editrice 2000, pag. 53.

48 Zaganelli Gioia, La Lettera del Prete Gianni, Luni Editrice 2000, pag. 55.

${ }^{49}$ Ibidem, pag. 55.
} 
e perisino nelle cronache gotiche ${ }^{50}$. Il successo interculturale di Gog e Magog sicuramente diede loro il primo posto nella lista dei residenti. Tra la buona associazione di animali, esseri straordinari e popoli, nel regno c'era un altro gruppo molto speciale - le dieci tribù disperse di Israele: „Al di là del fiume di pietre vivono le dieci tribù di Israele e nonostante esse si inventino dei loro re, sono in realtà soggette a noi e pagano tributi alla nostra Eccellenza"51.

La ricchezza della diversità degli abitanti del regno del Prete non fu certamente causale. L'autore della lettera era consapevole che collocare le convinzioni rilevanti per molte culture in un posto, avrebbe motivato un gran numero di persone a credere nel regno. Non sorprende, quindi, che la diffusione della lettera nel Medioevo fosse così vertiginosa: tutti potevano trovare in essa un pezzo della propria cultura, che dal momento che trovò un posto nel regno, doveva essere sicuramente importante anche per il potente sovrano.

La leggenda medievale sul regno perduto del Presbitero Gianni diventò l'ispirazione per molte generazioni di viaggiatori, cronisti e scrittori. A seconda del periodo, tuttavia, la visione di questa storia cambiava. Per la gente del Medioevo il regno era assolutamente reale e nessuno metteva in dubbio la sua esistenza. Era come parte dell'immagine del mondo - il mondo in cui Gianni aveva il proprio posto garantito. Se sia il Papa che i governanti credevano nell'esistenza di Giovanni, come poteva la gente umile non credere in lui? Chi avrebbe usato dire che il Papa aveva torto? E inoltre, c'era la questione della lettera: dopotutto, la missiva doveva avere un autore. Per la gente del Medioevo era indubbiamente il Presbitero. La lettera non perse il suo valore nemmeno nell'era delle scoperte geografiche in quanto era una sorta di prova dell'esistenza del regno misterioso. In effetti, l'unica prova. La missiva era l'unico tentativo tangibile da parte di Gianni di cantattare l'Occidente. L'invenzione della stampa aumentò la diffusione della lettera e tutti potevano scoprire quanto fosse straordinario il regno del Prete. Naturalmente le scoperte geografiche, e quindi l'approfondimento della conoscenza del mondo, conclusero finalmente la fase di ricerca di un paese inesistente. È interessante, tuttavia, notare che la fede nel regno è rimasta immutata per tanti secoli. Non c'è da stupirsi, quindi, che il tema dello stato situato nel paradiso terrestre abbia risvegliato e continuì a risvegliare l'immaginazione.

\footnotetext{
${ }^{50}$ Confronta: Iordanes Getica, un'opera conservata nelle note nelle opere degli altri autori.

${ }^{51}$ Zaganelli Gioia, La Lettera del Prete Gianni, Luni Editrice 2000, pag. 67.
} 


\section{Bibliografia}

Jacques le Goff (2017). Il cielo sceso in terra, Le radici medievali dell'Europa, Editori Laterza.

Giacomo de Vitry (1960). Lettres de Jacques de Vitry, (1160/70-1240), évêque de Saint-Jean d'Acre, éd. par R.B.C. Huygens, Leiden, E.J. Brill.

Guglielmo da Rubruck (2015). Itinerarium in Prester John: the Legend and its Source, Kegan Brewer, University of Sydney, Australia, Ashgate.

Helleiner Karl F. (1959). Preter John's Letter: A Medieval Utopia, Phoenix, vol. XIII-2.

Marco Polo (1991). Il Milione, Edizioni Studio Tesi.

Nowen Charies E. (1953). The Historical Prester John, Speculum XXVIII-3.

Ortalli G. (1997). Scenari e proposte per un Medioevo europeo, in Storia d'Europa, Einaudi, Torino.

Ottonis Episcopi Frisigensis (1912). Chronica sive historia de duabus civitatibus in Scriptores Rerum Germanicarum in usum scholarum ex monumentis Germaniae historicis separtim editi. Hannoverae et Lipsiae Impensis Bibliopolii Hahniani.

Peeters P. (1908). La martyrolge de Rabban Sliba, Analecta Bollandiana XXVII.

Radulphi de Coggeshall (1875). Chronicon anglicanum, De expugnatione Terræ Sanctæ libellus, Thomas Agnellus De morte et sepultura Henrici regis Angliæ junioris, Gesta Fulconis filii Warini, excerpta ex Otiis imperialibus Gervasii Tileburiensis. Ex codicibus manuscriptis edidit Josephus Stevenson $1875 \mathrm{r}$.

Zaganelli G. (2000). La lettera del Prete Gianni, Luni Editrice.

Zarncke F. (1980). Der Priester Johannes, Georg Olms Verlag Hildesheim, New York.

Zerncke F. (1996). Der Brief des Papstes Alexanders III an der Priester Johannes in Beckingham Ch., Hamilton B. Prester John, the Mongols and the Ten Lost Tribes; Ashgate Publishing Ltd.

\section{Fonti Internet}

https://histmag.org/Tajemnicze-krolestwo-ksiedza-Jana-1406; [dostęp 24.01.2019] 
